\title{
TiRS graphs and TiRS frames: a new setting for duals of canonical extensions
}

\author{
Andrew P.K. Craig, Maria J. Gouveia, and Miroslav Haviar \\ Dedicated to Professor Brian A. Davey on his 65th birthday
}

\begin{abstract}
We consider properties of the graphs that arise as duals of bounded lattices in Ploščica's representation via maximal partial maps into the two-element set. We introduce TiRS graphs, which abstract those duals of bounded lattices. We demonstrate their one-to-one correspondence with so-called TiRS frames, which are a subclass of the class of RS frames introduced by Gehrke to represent perfect lattices. This yields a dual representation of finite lattices via finite TiRS frames, or equivalently finite TiRS graphs, which generalises the well-known Birkhoff dual representation of finite distributive lattices via finite posets. By using both Ploščica's and Gehrke's representations in tandem, we present a new construction of the canonical extension of a bounded lattice. We present two open problems that will be of interest to researchers working in this area.
\end{abstract}

\section{Introduction}

The canonical extension $\mathbf{L}^{\delta}$ of a bounded lattice $\mathbf{L}$ was first introduced by Gehrke and Harding [12] as the complete lattice of Galois-closed sets associated with a polarity between the filter lattice $\operatorname{Filt}(\mathbf{L})$ and the ideal lattice $\operatorname{Idl}(\mathbf{L})$ of $\mathbf{L}$. We refer to Gehrke and Vosmaer [13] for a recent survey of the theory of canonical extensions for lattice-based algebras, including a discussion of their important role in the semantic modeling of logics. Important contributions to the theory of canonical and natural extensions of lattice-based algebras have also recently been made by Brian A. Davey et al. [6, 5, 8, 9] to whom the present paper is dedicated.

The variety $\mathcal{L}$ of bounded lattices is not finitely generated, thus no natural duality theory in the terms of Clark and Davey [1] is available for $\mathcal{L}$. There is a well-known representation for $\mathcal{L}$ due to Urquhart [15], which was later presented in the spirit of natural duality theory by Ploščica in [14]. In

Presented by R. Quackenbush.

Received October 31, 2013; accepted in final form September 9, 2014.

2010 Mathematics Subject Classification: Primary: 06B23; Secondary: 06D50, 06B15.

Key words and phrases: bounded lattice, canonical extension, perfect lattice, TiRS graph, TiRS frame, RS frame.

The first author gratefully acknowledges funding from the Claude Leon Foundation during the writing of this paper. He also acknowledges the support of the Rhodes Trust during his DPhil studies at Oxford when some of the preliminary work was done. The second author acknowledges support from Portuguese Project PEst-OE/MAT/UI0143/2014 of CAUL financed by FCT. The third author acknowledges support from Slovak grants VEGA 1/0212/13 and APVV-0223-10. 
[3], Ploščica's topological representation was used for constructing the canonical extension of a bounded lattice $\mathbf{L} \in \mathcal{L}$. Roughly speaking, this was done by modifying Priestley's representation for bounded distributive lattices $\mathbf{L}$ so that total maps from $\mathbf{L}$ into $\{0,1\}$ are replaced by appropriate maximallydefined partial maps into $\{0,1\}$, viewed either as a lattice or a partially ordered set. A partial homomorphism from $\mathbf{L}$ into the two-element lattice $\underline{\mathbf{2}}=(\{0,1\}, \wedge, \vee, 0,1)$ is a partial map $f: L \rightarrow\{0,1\}$ such that $\operatorname{dom} f$ is a bounded sublattice of $\mathbf{L}$ and the restriction $f \uparrow_{\operatorname{dom} f}$ is a bounded lattice homomorphism. A maximal partial homomorphism is a partial homomorphism with no proper extension. The set of maximal partial homomorphisms from $\mathbf{L}$ into $\underline{\mathbf{2}}$ is denoted by $\mathcal{L}^{\mathrm{mp}}(\mathbf{L}, \underline{\mathbf{2}})$. We observe that the elements of $\mathcal{L}^{\mathrm{mp}}(\mathbf{L}, \underline{\mathbf{2}})$ are in a one-to-one correspondence with the maximal disjoint filter-ideal pairs of $\mathbf{L}$.

More precisely now, Ploščica's dual of a lattice $\mathbf{L}$ is a graph with topology, $\mathrm{D}(\mathbf{L})=\left(\mathcal{L}^{\mathrm{mp}}(\mathbf{L}, \underline{\mathbf{2}}), E, \mathcal{T}\right)$, where the graph relation $E$ is given by $(f, g) \in E$ if and only if $f^{-1}(1) \cap g^{-1}(0)=\emptyset$ and the topology $\mathcal{T}$ has as a subbasis of closed sets the set $\left\{V_{a}, W_{a} \mid a \in L\right\}$, with $V_{a}=\left\{f \in \mathcal{L}^{\mathrm{mp}}(\mathbf{L}, \underline{\mathbf{2}}) \mid f(a)=0\right\}$ and $W_{a}=\left\{f \in \mathcal{L}^{\mathrm{mp}}(\mathbf{L}, \underline{\mathbf{2}}) \mid f(a)=1\right\}$. We will use the notation $\mathrm{D}^{b}(\mathbf{L})$ to refer to the graph $\left(\mathcal{L}^{\mathrm{mp}}(\mathbf{L}, \underline{\mathbf{2}}), E\right)$. (We note that what we will call a graph is usually referred to as a digraph.)

Given two graphs $\mathbf{X}=\left(X, E_{X}\right)$ and $\mathbf{Y}=\left(Y, E_{Y}\right)$, a maximal partial $E$-preserving map is a partial map $\varphi: X \rightarrow Y$ such that $\varphi$ preserves $E$ on its domain and any proper extension of $\varphi$ will not preserve $E$. The canonical extension of $\mathbf{L}$ constructed in [3] is then taken to be the lattice $\mathrm{C}\left(\mathrm{D}^{b}(\mathbf{L})\right)=\mathcal{G}^{\mathrm{mp}}\left(\mathrm{D}^{\mathrm{b}}(\mathbf{L}), \underset{\sim}{\mathbf{2}}\right)$ of all maximal partial $E$-preserving maps from $\mathrm{D}^{\mathrm{b}}(\mathbf{L})=\left(\mathcal{L}^{\mathrm{mp}}(\mathbf{L}, \underline{\mathbf{2}}), E\right)$ to the two-element graph $\underset{\sim}{\mathbf{2}}=(\{0,1\}, \leqslant)$. For these special graphs $\mathbf{X}=\mathrm{D}^{b}(\mathbf{L})$, as well as for arbitrary graphs $\mathbf{X}=(X, E)$, the lattice order in the (complete) lattice $\mathrm{C}(\mathbf{X})$ of all maximal partial $E$-preserving maps from $\mathbf{X}$ into $\underset{\sim}{2}$ is given by $\varphi \leqslant \psi$ iff $\varphi^{-1}(1) \subseteq \psi^{-1}(1)$ for all $\varphi, \psi \in \mathrm{C}(\mathbf{X})$ $($ see $[3,2])$.

In this paper, we show that duals of bounded lattices can be viewed as so-called TiRS graphs as well as so-called TiRS frames. In Section 2, TiRS graphs are introduced as an abstraction of the graphs $D^{b}(\mathbf{L})$ obtained from Ploščica's duals of bounded lattices $\mathbf{L}$. As the main result, a one-to-one correspondence between TiRS graphs and TiRS frames is shown. We note that TiRS frames are special RS frames; the latter were introduced by Gehrke [11] in her dual representation of so-called perfect lattices. We wish to emphasize that our correspondence between TiRS graphs and TiRS frames does not extend to a correspondence between RS graphs (which we introduce as a natural counterpart of the RS frames) and RS frames. Since they are in a one-to-one correspondence, we believe TiRS graphs and TiRS frames are better choices for classes of graphs and RS frames that generalise, respectively, the graphs and frames associated to bounded lattices.

In Section 3, we first prove that every finite RS frame is a TiRS frame and we point out that this yields a dual representation of finite lattices via 
finite TiRS frames, or equivalently finite TiRS graphs, which generalises the well-known Birkhoff dual representation of finite distributive lattices via finite posets. We then generalise the descriptions of the completely join-irreducible and the completely meet-irreducible elements in the complete lattices $\mathrm{C}(\mathbf{X})$, which were presented for the graphs $\mathbf{X}=\left(\mathcal{L}^{\mathrm{mp}}(\mathbf{L}, \underline{\mathbf{2}}), E\right)$ in [2], to arbitrary reflexive reduced graphs $\mathbf{X}=(X, E)$. Finally, we show that Ploščica's dual representation of bounded lattices and Gehrke's dual representation of perfect lattices can be used in tandem to provide a new construction of the canonical extension for an arbitrary bounded lattice.

For all concepts and results needed in this paper, we refer to Section 2 of [2], which can be used as our preliminaries and which is available online.

\section{TiRS graphs and TiRS frames}

In this section, we first show that bounded lattices give rise to particular graphs that we call TiRS graphs. More precisely, these graphs are an abstraction of the graphs $\mathrm{D}^{b}(\mathbf{L})$ obtained from Ploščica's duals of bounded lattices $\mathbf{L}$ by forgetting the topology. We also show how to carry the properties of TiRS graphs to the setting of RS frames introduced by Gehrke [11], and then how to associate to each TiRS graph a so-called TiRS frame in a one-to-one correspondence.

We start by identifying characteristics of the graph $\mathrm{D}^{\mathrm{b}}(\mathbf{L})=\left(\mathcal{L}^{\mathrm{mp}}(\mathbf{L}, \underline{\mathbf{2}}), E\right)$. Recall that $\mathcal{L}^{\mathrm{mp}}(\mathbf{L}, \underline{\mathbf{2}})$ is the set of maximal partial homomorphisms from $\mathbf{L}$ into $\underline{\mathbf{2}}$ and the relation $E$ is given by $f E g$ if and only if $f^{-1}(1) \cap g^{-1}(0)=\emptyset$.

From the well-known properties of filters and ideals of a bounded lattice and from the correspondence between elements $f \in \mathcal{L}^{\mathrm{mp}}(\mathbf{L}, \underline{\mathbf{2}})$ and maximal filter-ideal pairs $\left(f^{-1}(1), f^{-1}(0)\right)$, we obtain the following result.

Lemma 2.1. Let $\mathbf{L}$ be a bounded lattice and let $\mathbf{X}=\mathrm{D}^{b}(\mathbf{L})$. Then $E$ is reflexive and $\mathbf{X}$ satisfies the three conditions below:

(1) for every $f, g \in X$, if $f \neq g$, then $f^{-1}(1) \neq g^{-1}(1)$ or $f^{-1}(0) \neq g^{-1}(0)$;

(2) (i) for all $f, h \in X$, if $f^{-1}(1) \subsetneq h^{-1}(1)$, then $h^{-1}(1) \cap f^{-1}(0) \neq \emptyset$;

(ii) for all $g, h \in X$, if $g^{-1}(0) \subsetneq h^{-1}(0)$, then $g^{-1}(1) \cap h^{-1}(0) \neq \emptyset$;

(3) for all $f, g \in X$, if $f^{-1}(1) \cap g^{-1}(0)=\emptyset$, then there exists $h \in X$ such that $f^{-1}(1) \subseteq h^{-1}(1)$ and $g^{-1}(0) \subseteq h^{-1}(0)$.

Proof. (1): This is clear.

(2): If $f^{-1}(1) \subsetneq h^{-1}(1)$ for $f, h \in X$, then $h^{-1}(1) \cap f^{-1}(0) \neq \emptyset$, because $f^{-1}(1)$ is a maximal filter with respect to not intersecting $f^{-1}(0)$. This shows (i), while (ii) can be derived analogously.

(3): If $f^{-1}(1) \cap g^{-1}(0)=\emptyset$ for $f, g \in X$, then by Zorn's Lemma, the filterideal pair $\left(f^{-1}(1), g^{-1}(0)\right)$ can be extended to a maximal one, so there exists $h \in X$ for which $f^{-1}(1) \subseteq h^{-1}(1)$ and $g^{-1}(0) \subseteq h^{-1}(0)$. 
For a graph $\mathbf{X}=(X, E)$ and $x \in X$, we denote by the symbols $x E$ and $E x$ the sets $\{y \in X \mid(x, y) \in E\}$ and $\{y \in X \mid(y, x) \in E\}$, respectively. We note that for $f, g \in \mathcal{L}^{\mathrm{mp}}(\mathbf{L}, \underline{\mathbf{2}})$, we have that

$$
f^{-1}(1) \subseteq g^{-1}(1) \Longleftrightarrow g E \subseteq f E \quad \text { and } \quad f^{-1}(0) \subseteq g^{-1}(0) \Longleftrightarrow E g \subseteq E f,
$$

and consequently, conditions (1) to (3) can be rewritten by using the notation $x E$ and $E x$ with $x \in \mathcal{L}^{\mathrm{mp}}(\mathbf{L}, \underline{\mathbf{2}})$. Motivated by the graphs $\mathrm{D}^{b}(\mathbf{L})$ above, we now generalise the properties (1), (2), and (3) to arbitrary graphs $\mathbf{X}=(X, E)$ as follows:

(S) for every $x, y \in X$, if $x \neq y$, then $x E \neq y E$ or $E x \neq E y$;

(R) (i) for all $x, z \in X$, if $z E \subsetneq x E$, then $(z, x) \notin E$;

(ii) for all $y, z \in X$, if $E z \subsetneq E y$, then $(y, z) \notin E$;

(Ti) for all $x, y \in X$, if $(x, y) \in E$, then there exists $z \in X$ such that $z E \subseteq x E$ and $E z \subseteq E y$.

When $E$ is also reflexive, it is easily seen that the condition (Ti) can equivalently be written as follows:

$(\mathrm{Ti})^{\prime}$ for all $x, y \in X$, if $(x, y) \in E$, then there exists $z$ such that $(x, z) \in E$ and $(z, y) \in E$, and for every $w \in X,(z, w) \in E$ implies $(x, w) \in E$ and $(w, z) \in E$ implies $(w, y) \in E$.

If $E$ were a partial order, we would say that the elements $z$ were in the interval $[x, y]$. For the elements $z$, we will use the term transitive interval elements (with respect to $(x, y) \in E$ ). Hence, condition (Ti) guarantees the existence of transitive interval elements for every $x, y \in X$ such that $(x, y) \in E$. So we shall call (Ti) the transitive interval element condition.

Definition 2.2. A graph $\mathbf{X}=(X, E)$ is:

(1) a separated graph if it satisfies condition $(\mathrm{S})$;

(2) a reduced graph if it satisfies condition $(\mathrm{R})$;

(3) an $R S$ graph if it satisfies conditions ( $\mathrm{R})$ and $(\mathrm{S})$;

(4) a TiRS graph if $E$ is reflexive and $\mathbf{X}$ satisfies conditions (R), (S) and (Ti).

Hence, using Lemma 2.1, the next statement follows immediately.

Proposition 2.3. For any bounded lattice $\mathbf{L}$, its dual graph $\mathbf{X}=\mathrm{D}^{\mathrm{b}}(\mathbf{L})$ is a TiRS graph.

A natural question to ask now is: which TiRS graphs arise as duals of bounded lattices? In the case of bounded distributive lattices, this question reduces to the question of which posets arise as duals of bounded distributive lattices in the Priestley duality. As is well known, this question of representable posets is extremely hard (see for example the paper by Davey [4]). In our situation, we know that there are TiRS graphs that are not the duals of bounded lattices. Examples are any non-representable posets, as any poset is automatically a TiRS graph. We expect that the question above regarding the non-distributive case will be difficult and we pose this as an open problem: 
Problem 1. Which TiRS graphs arise as duals of bounded lattices?

Ploščica [14, Section 3] noted that there were cases where the graph of maximal partial homomorphisms from the bounded lattice $\mathbf{L}$ into $\underline{\mathbf{2}}$ was not minimal amongst those graphs with topology that could serve as a representation of $\mathbf{L}$. An example of this is presented in Figure 1, where $\mathbf{L}$ is taken to be the modular lattice $\mathbf{M}_{3}$ and the graph $\mathbf{Y}$ is a minimal graph. The elements of the graphs $\mathbf{X}$ and $\mathbf{Y}$ are described as follows: the elements of $X$ are the maximal partial homomorphisms $f_{u v}$, with $u, v \in \mathbf{L} \backslash\{0,1\}$, defined by $f_{u v}^{-1}(1)=\uparrow u$ and $f_{u v}^{-1}(0)=\downarrow v$. The graph $\mathbf{Y}$ is the subgraph of $X$ whose vertex set is $Y=\left\{f_{a b}, f_{b c}, f_{c a}\right\}$. It is straightforward to check that the lattice $\mathbf{L}$ is isomorphic to the lattice $\mathbf{G}_{\mathcal{T}}^{\mathrm{mp}}\left(\mathbf{Y}_{\mathcal{T}}, \mathbf{2}_{\mathcal{T}}\right)$ via the isomorphism $a \mapsto e_{a}$. We note that because the lattice $\mathbf{L}$ is finite, it makes no difference whether we consider $\mathcal{G}_{\mathcal{T}}^{\mathrm{mp}}\left(\mathbf{Y}_{\mathcal{T}}, \underset{\sim}{\mathbf{2}} \mathcal{T}\right.$ ) or $\boldsymbol{G}^{\mathrm{mp}}(\mathbf{Y}, \underset{\sim}{\mathbf{2}}$ ) (and similarly for $\mathbf{X}$ ).

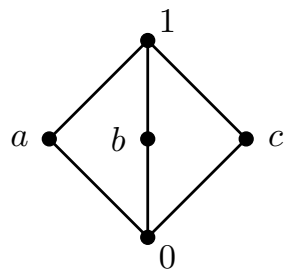

$\mathbf{L}$

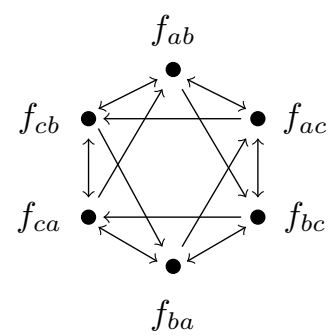

$\mathbf{X}$

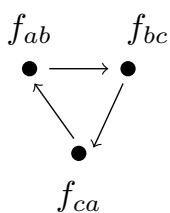

$\mathbf{Y}$

Figure 1. The modular lattice $\mathbf{M}_{3}$ and two of its graph representations $\mathbf{X}=\mathrm{D}^{b}(\mathbf{L})$ and $\mathbf{Y}$.

The graph $\mathbf{X}$ in Figure 1 is a TiRS graph (this is the content of Proposition 2.3) while the RS graph $\mathbf{Y}$ does not satisfy the condition (Ti).

Given a bounded lattice $\mathbf{L}$, the TiRS graph $\mathbf{X}=\mathrm{D}^{b}(\mathbf{L})$ is naturally associated to the structure $\mathbf{F}:=\left(X_{1}, X_{2}, R\right)$, where $X_{1}$ is the set of all filters $f^{-1}(1)$ of $\mathbf{L}$ with $f \in X$, and $X_{2}$ is the set of all ideals $g^{-1}(0)$ of $\mathbf{L}$ with $g \in X$. The binary relation $R \subseteq X_{1} \times X_{2}$ is defined by

$$
\left(f^{-1}(1), g^{-1}(0)\right) \in R \quad \text { if } \quad f^{-1}(1) \cap g^{-1}(0) \neq \emptyset,
$$

or equivalently (henceforth we will write the relation symbol $R$ in the middle),

$$
f^{-1}(1) R g^{-1}(0) \quad \text { if } \quad(f, g) \notin E .
$$

Note that $X_{1}$ and $X_{2}$ are respectively the set of all filters and the set of all ideals in maximal filter-ideal pairs.

Let us recall that a structure $\left(X_{1}, X_{2}, R\right)$, where $X_{1}$ and $X_{2}$ are non-empty sets and $R \subseteq X_{1} \times X_{2}$, is said to be a frame. For $x \in X_{1}$ and $y \in X_{2}$, we define

$$
x R:=\left\{y \in X_{2} \mid x R y\right\} \text { and } R y:=\left\{x \in X_{1} \mid x R y\right\} .
$$


For an arbitrary frame $\mathbf{F}=\left(X_{1}, X_{2}, R\right)$ we now define conditions $(\mathrm{S})$ and $(\mathrm{R})$ as follows:

(S) for all $x_{1}, x_{2} \in X_{1}$ and $y_{1}, y_{2} \in X_{2}$,

(i) $x_{1} \neq x_{2}$ implies $x_{1} R \neq x_{2} R$;

(ii) $y_{1} \neq y_{2}$ implies $R y_{1} \neq R y_{2}$.

(R) (i) for every $x \in X_{1}$, there exists $y \in X_{2}$ such that $\neg(x R y)$ and $\forall w \in X_{1}((w \neq x \quad \& \quad x R \subseteq w R) \Rightarrow w R y)$

(ii) for every $y \in X_{2}$, there exists $x \in X_{1}$ such that $\neg(x R y)$ and $\forall z \in X_{2}((z \neq y \quad \& \quad R y \subseteq R z) \Rightarrow x R z)$.

The frames that satisfy the conditions $(\mathrm{R})$ and $(\mathrm{S})$ are called reduced separated frames, or RS frames for short, and were introduced by Gehrke [11]. They were formulated as a two-sorted generalisation of Kripke frames to be used for relational semantics of substructural logics.

Here we introduce the $(\mathrm{Ti})$ condition for frames. It is motivated by the $(\mathrm{Ti})$ condition on graphs:

(Ti) for every $x \in X_{1}$ and for every $y \in X_{2}$, if $\neg(x R y)$, then there exist $w \in X_{1}$ and $z \in X_{2}$ such that

(i) $\neg(w R z)$;

(ii) $x R \subseteq w R$ and $R y \subseteq R z$;

(iii) for every $u \in X_{1}$, if $u \neq w$ and $w R \subseteq u R$, then $u R z$;

(iv) for every $v \in X_{2}$, if $v \neq z$ and $R z \subseteq R v$, then $w R v$.

Definition 2.4. A TiRS frame is a frame $\left(X_{1}, X_{2}, R\right)$ that satisfies conditions $(\mathrm{R}),(\mathrm{S})$, and $(\mathrm{Ti})$, i.e., it is an RS frame that satisfies condition (Ti).

The following is an example of an RS frame that is not TiRS. Let $X_{1}=$ $\left\{a_{i}\right\}_{i \in \omega}, X_{2}=\left\{b_{i}\right\}_{i \in \omega}$ and

$$
R=\left\{\left(a_{1}, b_{0}\right),\left(a_{0}, b_{1}\right)\right\} \cup\left\{\left(a_{i}, b_{j}\right) \mid 2 \leqslant i, 1 \leqslant j \leqslant i\right\} .
$$

By considering $\neg\left(a_{0} R b_{0}\right)$, it is straightforward to show that $\left(X_{1}, X_{2}, R\right)$ does not satisfy $(\mathrm{Ti})$.

We observe that for the frame $\mathbf{F}:=\left(X_{1}, X_{2}, R\right)$ naturally associated to the TiRS graph $\mathrm{D}^{b}(\mathbf{L})$ dual to some bounded lattice $\mathbf{L}$, not every element $F \in X_{1}$ need determine a unique maximal partial homomorphism $f \in \mathcal{L}^{\mathrm{mp}}(\mathbf{L}, \underline{\mathbf{2}})$ since $F$ might be the filter of more than one maximal filter-ideal pair. However, every $F$ determines a unique class of elements $f \in \mathcal{L}^{\mathrm{mp}}(\mathbf{L}, \underline{\mathbf{2}})$ such that $f^{-1}(1)=$ $F$. This gives rise to an equivalence relation $\sim_{1}$ on $\mathcal{L}^{\mathrm{mp}}(\mathbf{L}, \underline{\mathbf{2}})$ for which $X_{1}$ may be identified with the quotient $\mathcal{L}^{\mathrm{mp}}(\mathbf{L}, \underline{\mathbf{2}}) / \sim_{1}$. Similarly, we may define on $\mathcal{L}^{\mathrm{mp}}(\mathbf{L}, \underline{\mathbf{2}})$ an equivalence relation $\sim_{2}$ by $f \sim_{2} g$ iff $f^{-1}(0)=g^{-1}(0)$; this allows us to identify $X_{2}$ with the quotient $\mathcal{L}^{\mathrm{mp}}(\mathbf{L}, \underline{\mathbf{2}}) / \sim_{2}$. We note that $f^{-1}(1)=g^{-1}(1)$ is equivalent to $f E=g E$ while $f^{-1}(0)=g^{-1}(0)$ is equivalent to $E f=E g$. This motivates us to define equivalence relations on $X$ and to define sets $X_{1}$ and $X_{2}$ in order to associate a frame $\left(X_{1}, X_{2}, R\right)$ to a graph without assuming the graph is $\mathrm{D}^{b}(\mathbf{L})$ for some lattice $\mathbf{L}$. 
Definition 2.5. Let $\mathbf{X}=(X, E)$ be a graph. The associated frame $\rho(\mathbf{X})$ is the frame $\left(X_{1}, X_{2}, R_{\rho(\mathbf{X})}\right)$ where

(i) $X_{1}=X / \sim_{1}$ for the equivalence relation $\sim_{1}$ on $X$ given by

$$
x \sim_{1} y \text { if } x E=y E ;
$$

(ii) $X_{2}=X / \sim_{2}$ for the equivalence relation $\sim_{2}$ on $X$ given by

$$
x \sim_{2} y \text { if } E x=E y
$$

(iii) $R_{\rho(\mathbf{X})}$ is the relation given by

$$
[x]_{1} R_{\rho(\mathbf{X})}[y]_{2} \Longleftrightarrow(x, y) \notin E,
$$

where $[x]_{1}$ and $[y]_{2}$ are, respectively, the $\sim_{1}$-equivalence class of $x$ and the $\sim_{2}$-equivalence class of $y$.

We omit the subscript $\rho(\mathbf{X})$ in $R_{\rho(\mathbf{X})}$ whenever it is clear to which relation $R$ refers.

Now we prove that the frames $\rho(\mathbf{X})$ associated to TiRS graphs $\mathbf{X}$ are necessarily TiRS.

Proposition 2.6. If $\mathbf{X}=(X, E)$ is a TiRS graph, then the associated frame $\rho(\mathbf{X})=\left(X_{1}, X_{2}, R_{\rho(\mathbf{X})}\right)$ is a TiRS frame.

Proof. (S): Let $x, y \in X$ and assume that $[x]_{1} \neq[y]_{1}$. Then $x E \neq y E$, and so $x E \not \subset y E$ or $y E \nsubseteq \subseteq x E$. Without loss of generality, we may assume that there exists $z \in X$ such that $(x, z) \in E$ but $(y, z) \notin E$. Now $[y]_{1} R[z]_{2}$ but $\neg\left([x]_{1} R[z]_{2}\right)$, and so $[x]_{1} R \neq[y]_{1} R$. Thus, (S)(i) holds. Similarly, we prove that $\rho(\mathbf{X})$ satisfies $(\mathrm{S})(\mathrm{ii})$.

$(\mathrm{R})$ : Let $[x]_{1} \in X_{1}$ and consider $[x]_{2} \in X_{2}$. Since $(x, x) \in E$, we have that $\neg\left([x]_{1} R[x]_{2}\right)$. Let $[w]_{1} \in X_{1}$ and assume that $[w]_{1} \neq[x]_{1}$ and $[x]_{1} R \subseteq$ $[w]_{1} R$. Note that $[x]_{1} R \subseteq[w]_{1} R$ implies $w E \subseteq x E$. Since $w E \neq x E$, we have $w E \subsetneq x E$. But $\mathbf{X}$ satisfies the corresponding condition (R)(i) for graphs, and so $(w, x) \notin E$, which implies that $[w]_{1} R[x]_{2}$. Thus, (R)(i) holds for $\rho(\mathbf{X})$. Similarly, we prove that $\rho(\mathbf{X})$ satisfies $(\mathrm{R})(\mathrm{ii})$.

(Ti): Let $[x]_{1} \in X_{1}$ and $[y]_{2} \in X_{2}$, and assume that $\neg\left([x]_{1} R[y]_{2}\right)$. Since $\mathbf{X}$ is a TiRS graph and $(x, y) \in E$, there exists $z \in X$ such that $z E \subseteq x E$ and $E z \subseteq E y$. Then $[z]_{1} \in X_{1},[z]_{2} \in X_{2},[x]_{1} R \subseteq[z]_{1} R, R[y]_{2} \subseteq R[z]_{2}$, and by the reflexivity of $E, \neg\left([z]_{1} R[z]_{2}\right)$. Moreover, if $u \in X$ satisfies $[u]_{1} \neq[z]_{1}$ and $[z]_{1} R \subseteq[u]_{1} R$, then $u E \subsetneq z E$, which implies $(u, z) \notin E$ by condition (R)(i) for graphs. Thus, $[u]_{1} R[z]_{2}$, finishing the proof of condition (Ti)(iii). The proof of $(\mathrm{Ti})(\mathrm{iv})$ is analogous.

From Propositions 2.3 and 2.6, it follows now that the frame $\rho\left(\mathrm{D}^{\mathrm{b}}(\mathbf{L})\right)$ associated to a bounded lattice $\mathbf{L}$ is TiRS.

Corollary 2.7. Let $\mathbf{L}$ be a bounded lattice, let $\mathbf{X}=\mathrm{D}^{b}(\mathbf{L})$ be its dual TiRS graph, and let $\rho\left(\mathrm{D}^{b}(\mathbf{L})\right)$ be its associated frame. Then $\rho\left(\mathrm{D}^{b}(\mathbf{L})\right)$ is a TiRS frame. 
Next we show that, conversely, it is possible to assign a graph to every TiRS frame in such a way that we get a correspondence between TiRS graphs and TiRS frames. Moreover, we would like that this correspondence allow us to recapture every TiRS graph $\mathbf{X}$ from its corresponding frame $\rho(\mathbf{X})$.

Once again, we start by considering the particular case when $\mathbf{X}$ is the graph $\mathrm{D}^{b}(\mathbf{L})$ for some bounded lattice $\mathbf{L}$. We recall that the frame $\rho(\mathbf{X})$ can be taken so that $X_{1}$ and $X_{2}$ are, respectively, the set of filters $f^{-1}(1)$ and the set of ideals $f^{-1}(0)$, with $f \in \mathcal{L}^{\mathrm{mp}}(\mathbf{L}, \underline{\mathbf{2}})$. We also recall that the elements $f \in \mathcal{L}^{\mathrm{mp}}(\mathbf{L}, \underline{\mathbf{2}})$ are completely determined by the pairs $\left(f^{-1}(1), f^{-1}(0)\right)$, which are exactly the pairs $(F, I)$ such that $F \cap I=\emptyset, F$ is a filter that is maximal with respect to not intersecting $I$, and $I$ is an ideal that is maximal with respect to not intersecting $F$. Hence, to recapture $\mathbf{X}$, we just need to take the appropriate pairs $(x, y)$ of $X_{1} \times X_{2}$, those given by condition (Ti). We note that the nonemptyness of the sets $X_{1}$ and $X_{2}$ of a frame allied to condition (R) implies the existence of pairs $(x, y)$ such that $\neg(x R y)$, and consequently, in a TiRS frame the existence of pairs $(w, z)$ that satisfy (Ti) (i),(iii), and (iv) is guaranteed. This leads us to the next definition.

Definition 2.8. Let $\mathbf{F}=\left(X_{1}, X_{2}, R\right)$ be a TiRS frame. The associated graph $\operatorname{gr}(\mathbf{F})$ is $\left(H_{\mathbf{F}}, K_{\mathbf{F}}\right)$, where the vertex set $H_{\mathbf{F}}$ is the subset of $X_{1} \times X_{2}$ of all pairs $(x, y)$ that satisfy the following conditions:

(a) $\neg(x R y)$;

(b) for every $u \in X_{1}$, if $u \neq x$ and $x R \subseteq u R$, then $u R y$;

(c) for every $v \in X_{2}$, if $v \neq y$ and $R y \subseteq R v$, then $x R v$.

The edge set $K_{\mathbf{F}}$ is formed by the pairs $((x, y),(w, z))$ such that $\neg(x R z)$.

We omit the subscript $\mathbf{F}$ in $H_{\mathbf{F}}$ and in $K_{\mathbf{F}}$ whenever it is clear which vertex set and edge set we refer to.

Lemma 2.9. Let $\mathbf{F}=\left(X_{1}, X_{2}, R\right)$ be a TiRS frame.

(i) For every $x \in X_{1}$, there exists $y \in X_{2}$ such that $(x, y) \in H$.

(ii) For every $y \in X_{2}$, there exists $x \in X_{1}$ such that $(x, y) \in H$.

(iii) If $(x, y),(w, z) \in H$, then

$$
(w, z) K \subseteq(x, y) K \Leftrightarrow x R \subseteq w R \quad \& \quad K(w, z) \subseteq K(x, y) \Leftrightarrow R y \subseteq R z .
$$

Proof. (i): Let $x \in X_{1}$. By hypothesis, $\mathbf{F}$ satisfies condition (R) for frames, which implies the existence of $v \in X_{2}$ such that $\neg(x R v)$ and

$$
\text { for every } u \in X_{1} \text {, if } u \neq x \text { and } x R \subseteq u R \text {, then } u R v \text {. }
$$

By condition ( $\mathrm{Ti}$ ) for frames, there exists $(w, z) \in H$ such that $x R \subseteq w R$ and $R v \subseteq R z$. Since $\neg(w R z)$ and $R v \subseteq R z$, we must have $\neg(w R v)$. But then $(*)$ implies $w=x$ and consequently $(x, z) \in H$. Thus, (i) holds.

(ii): The proof is similar. 
(iii): Consider $(x, y),(w, z) \in H$. By (ii), for every element $v \in X_{2}$, there exists $u \in X_{1}$ such that $(u, v) \in H$. Hence, the following holds:

$$
\begin{aligned}
& (w, z) K \subseteq(x, y) K \\
\Longleftrightarrow & \{(u, v) \in H \mid((x, y),(u, v)) \notin K\} \subseteq\{(u, v) \in H \mid((w, z),(u, v)) \notin K\} \\
\Longleftrightarrow & \{(u, v) \in H \mid(x, v) \in R\} \subseteq\{(u, v) \in H \mid(w, v) \in R\} \\
\Longleftrightarrow & \left\{v \in X_{2} \mid(x, v) \in R\right\} \subseteq\left\{v \in X_{2} \mid(w, v) \in R\right\} \\
\Longleftrightarrow & x R \subseteq w R .
\end{aligned}
$$

Similarly, one can prove that $K(w, z) \subseteq K(x, y) \Longleftrightarrow R y \subseteq R z$.

Proposition 2.10. If $\mathbf{F}=\left(X_{1}, X_{2}, R\right)$ is a TiRS frame, then its associated graph $\operatorname{gr}(\mathbf{F})$ is a TiRS graph.

Proof. (S): Let us consider pairs $(x, y),(w, z) \in H$ such that $(x, y) \neq(w, z)$. Without loss of generality, assume that $x \neq w$. Since $\mathbf{F}$ satisfies $(\mathrm{S})$ for frames, we have that $x R \neq w R$, and therefore $x R \nsubseteq w R$ or $w R \nsubseteq x R$. Again without loss of generality, assume that $x R \nsubseteq w R$. Then there exists $v \in X_{2}$ such that $x R v$ but $\neg(w R v)$. By Lemma 2.9(ii), there exists $u \in X_{1}$ such that $(u, v) \in H$. Then $((x, y),(u, v)) \notin K$ but $((w, z),(u, v)) \in K$, and hence $(x, y) K \neq(w, z) K$.

(R): For (i), consider $(x, y),(w, z) \in H$ and assume that $(w, z) K \subsetneq(x, y) K$. It follows by Lemma 2.9(iii) that $x R \subseteq w R$ and $x$ and $w$ must be distinct, because $x=w$ would imply $w R \subseteq x R$, and so $(w, z) K=(x, y) K$. Since $(x, y) \in H, w \neq x$, and $x R \subseteq w R$, we have $w R y$ by condition (b) of Definition 2.8. Thus, $((w, z),(x, y)) \notin K$. One can analogously prove $\mathrm{R}$ (ii).

(Ti): Take $(x, y),(w, z) \in H$ with $((x, y),(w, z)) \in K$, that is, $\neg(x R z)$. By condition (Ti) for frames, there exist $u \in X_{1}$ and $v \in X_{2}$ such that $(u, v) \in H$, $x R \subseteq u R$, and $R z \subseteq R v$. Finally, we note that $x R \subseteq u R$ and $R z \subseteq R v$ imply $(u, v) K \subseteq(x, y) K$ and $K(u, v) \subseteq K(w, z)$, by Lemma 2.9(iii).

Definition 2.11. Two graphs $\mathbf{X}=\left(X, E_{X}\right)$ and $\mathbf{Y}=\left(Y, E_{Y}\right)$ are isomorphic (denoted $\mathbf{X} \simeq \mathbf{Y}$ ) if there exists a bijective map $\alpha: X \rightarrow Y$ such that

$$
\forall x_{1}, x_{2} \in X \quad\left(x_{1}, x_{2}\right) \in E_{X} \Longleftrightarrow\left(\alpha\left(x_{1}\right), \alpha\left(x_{2}\right)\right) \in E_{Y},
$$

and we refer to such a map as the graph-isomorphism $\alpha: \mathbf{X} \rightarrow \mathbf{Y}$.

Two frames $\mathbf{F}=\left(X_{1}, X_{2}, R_{F}\right)$ and $\mathbf{G}=\left(Y_{1}, Y_{2}, R_{G}\right)$ are isomorphic (denoted $\mathbf{F} \simeq \mathbf{G}$ ) if there exists a pair $\left(\beta_{1}, \beta_{2}\right)$ of bijective maps $\beta_{i}: X_{i} \rightarrow Y_{i}$ (for $i=1,2)$ with

$$
\forall x_{1} \in X_{1} \forall x_{2} \in X_{2} \quad\left(x_{1} R_{F} x_{2} \Longleftrightarrow \beta_{1}\left(x_{1}\right) R_{G} \beta_{2}\left(x_{2}\right)\right),
$$

and we refer to such a pair as the frame-isomorphism $\left(\beta_{1}, \beta_{2}\right): \mathbf{F} \rightarrow \mathbf{G}$.

Let $\mathbf{F}=\left(X_{1}, X_{2}, R\right)$ be a TiRS frame. We observe that for every $x \in X_{1}$, there exists $y \in X_{2}$ such that $(x, y) \in H$, by Lemma 2.9, and for every $z \in X_{2}$ satisfying $(x, z) \in H$, we have $[(x, y)]_{1}=[(x, z)]_{1}$. Hence, we may define a $\operatorname{map} \beta_{1}: X_{1} \rightarrow H / \sim_{1}$ by $\beta_{1}(x)=[(x, y)]_{1}$, where $y \in X_{2}$ satisfies $(x, y) \in H$. 
Similarly, we may define a map $\beta_{2}: X_{2} \rightarrow H / \sim_{2}$ by $\beta_{2}(y)=[(x, y)]_{2}$, where $x \in X_{1}$ satisfies $(x, y) \in H$. We define $\beta_{F}$ to be the pair $\left(\beta_{1}, \beta_{2}\right)$. As we will see later, the pair $\beta_{F}$ is a frame-isomorphism.

Lemma 2.12. Let $\mathbf{X}=(X, E)$ be a TiRS graph. The elements of $H_{\rho(\mathbf{X})}$ are exactly the pairs $\left([x]_{1},[x]_{2}\right)$, with $x \in X$.

Proof. For every $x \in X$, the reflexivity of $E$ and condition (R) satisfied by $\mathbf{X}$ guarantee that the pair $\left([x]_{1},[x]_{2}\right)$ belongs to $H$. Let $x, y \in X$ and assume that $\left([x]_{1},[y]_{2}\right) \in H$. Then $\neg\left([x]_{1} R[y]_{2}\right)$. Since $(x, y) \in E$ and $\mathbf{X}$ satisfies (Ti), there exists $z \in X$ such that $z E \subseteq x E$ and $E z \subseteq E y$, and (as we can see from $\left.(\mathrm{Ti})^{\prime}\right),(x, z),(z, y) \in E$. From $z E \subseteq x E$ and $(z, y) \in E$, we obtain $[x]_{1} R \subseteq[z]_{1} R$ and $\neg\left([z]_{1} R[y]_{2}\right)$. But then $\left([x]_{1},[y]_{2}\right) \in H$ implies $[x]_{1}=[z]_{1}$. Similarly, from $E z \subseteq E y$ and $(x, z) \in E$, we conclude $[y]_{2}=[z]_{2}$. Thus, $\left([x]_{1},[y]_{2}\right)=\left([z]_{1},[z]_{2}\right)$.

For a TiRS graph $\mathbf{X}=(X, E)$, the previous lemma allows us to define a map $\alpha_{X}: X \rightarrow \operatorname{gr}(\rho(\mathbf{X}))$ by $\alpha_{X}(x)=\left([x]_{1},[x]_{2}\right)$. In the proof of the following result, we show that $\alpha_{X}$ is a graph isomorphism. This result shows that the established correspondence between TiRS graphs and TiRS frames is one-toone.

Theorem 2.13. Let $\mathbf{X}=(X, E)$ be a TiRS graph and $\mathbf{F}=\left(X_{1}, X_{2}, R\right)$ be a TiRS frame. Then

(a) the graphs $\mathbf{X}$ and $\operatorname{gr}(\rho(\mathbf{X}))$ are isomorphic;

(b) the frames $\mathbf{F}$ and $\rho(\operatorname{gr}(\mathbf{F}))$ are isomorphic.

Proof. (a): For $x, y \in X$, we have that $\left([x]_{1},[x]_{2}\right)=\left([y]_{1},[y]_{2}\right)$ implies $x E=$ $y E$ and $E x=E y$. By $(\mathrm{S})$, this implies $x=y$. Hence, the map $\alpha_{X}$ is one-toone, and by Lemma 2.12 , it is also onto. Finally, we note that we have

$$
(x, y) \in E \quad \text { iff } \quad \neg\left([x]_{1} R[y]_{2}\right) \quad \text { iff } \quad\left(\left([x]_{1},[x]_{2}\right),\left([y]_{1}[y]_{2}\right)\right) \in K .
$$

(b): For $x \in X_{1}$ and $y \in X_{2}$, we take $w \in X_{1}$ and $z \in X_{2}$ with $(x, z),(w, y) \in$ $H$. Then $x R y$ iff $((x, z),(w, y)) \notin K$ iff $[(x, z)]_{1} R[(w, y)]_{2}$ iff $\beta_{1}(x) R \beta_{2}(y)$, where $\beta_{1}$ and $\beta_{2}$ are the maps that satisfy $\beta_{F}=\left(\beta_{1}, \beta_{2}\right)$. Finally, we need to prove that the maps $\beta_{1}$ and $\beta_{2}$ are one-to-one and $\beta_{1}\left(X_{1}\right)=H / \sim_{1}$ and $\beta_{2}\left(X_{2}\right)=H / \sim_{2}$. Let $x, w \in X_{1}$ and assume that $\beta_{1}(x)=\beta_{1}(w)$, i.e., $[(x, y)]_{1}=[(w, z)]_{1}$ for some $y, z \in X_{2}$ such that $(x, y),(w, z) \in H$. Then $x R=w R$, which, by condition $(\mathrm{S})$ for frames, implies $x=w$. Hence, $\beta_{1} \uparrow_{X_{1}}$ is one-to-one. Similarly, $\beta_{2} \Upsilon_{X_{2}}$ is one-to-one. Now take $[(x, y)]_{1} \in H / \sim_{1}$ with $(x, y) \in H$. Then $\beta_{1}(x)=[(x, y)]_{1}$, and so $\beta_{1} \uparrow_{X_{1}}$ is onto. Similarly, $\beta_{2} \uparrow_{X_{2}}$ is onto. Therefore, $\beta_{F}$ is a frame-isomorphism.

\section{Canonical extensions of bounded lattices and TiRS graphs}

In the previous section, motivated by graphs coming from Ploščica's representation of bounded lattices, we introduced TiRS graphs and showed how 
these structures correspond to particular RS frames that we call TiRS frames. The work of Dunn, Gehrke and Palmigiano [10, Section 4] and Gehrke [11, Section 2] combine to provide a dual representation between RS frames and so-called perfect lattices. Let us recall that a complete lattice $\mathbf{C}$ is said to be perfect if the set of its completely join-irreducible elements, $J^{\infty}(\mathbf{C})$, is join-dense and the set of its completely meet-irreducible elements, $M^{\infty}(\mathbf{C})$, is meet-dense in the lattice. Gehrke and Harding [12, Lemma 3.4] showed that $J^{\infty}\left(\mathbf{L}^{\delta}\right)$ is join-dense in $\mathbf{L}^{\delta}$ and that $M^{\infty}\left(\mathbf{L}^{\delta}\right)$ is meet-dense in $\mathbf{L}^{\delta}$. Hence, the canonical extension of a bounded lattice is a perfect lattice, and so the dual representation between RS frames and perfect lattices provides insights into the theory of canonical extensions for arbitrary bounded lattices. As we proved in [3, Theorem 3.11], the canonical extension of a bounded lattice $\mathbf{L}$ can be taken to be $\mathrm{C}(\mathbf{X})=\mathcal{G}^{\mathrm{mp}}(\mathbf{X}, \underset{\sim}{\mathbf{2}})$, where $\mathbf{X}=\left(\mathcal{L}^{\mathrm{mp}}(\mathbf{L}, \underline{\mathbf{2}}), E\right)$ is the TiRS graph dual to $\mathbf{L}$.

We start this section by showing that every finite RS frame is in fact a TiRS frame. The graph $\mathbf{Y}$ in Figure 1 showed that, in contrast to this, the classes of finite RS graphs and finite TiRS graphs are not the same. It follows that our correspondence between TiRS graphs and TiRS frames does not extend to a correspondence between RS graphs and RS frames.

Proposition 3.1. Every finite separated frame satisfies (Ti). Consequently, every finite $R S$ frame is a TiRS frame.

Proof. Let $F=\left(X_{1}, X_{2}, R\right)$ be a frame that satisfies (S). Let $x \in X_{1}, y \in X_{2}$, and assume that $\neg(x R y)$. Define

$$
S_{1}:=\left\{u \in X_{1} \mid x R \subseteq u R, \neg(u R y)\right\} .
$$

Since $x \in S_{1}$ and $X_{1}$ is finite, the set $T_{1}=\left\{u R \mid u \in S_{1}\right\}$ is non-empty and finite. Thus, $T_{1}$ has a maximal element (with respect to the inclusion order). Let $w \in S_{1}$ be such that $w R$ is a maximal element of $T_{1}$.

Now define

$$
S_{2}:=\left\{v \in X_{2} \mid R y \subseteq R v, \neg(w R v)\right\} .
$$

Since $y \in S_{2}$ and $X_{2}$ is finite, the set $T_{2}=\left\{R v \mid v \in S_{2}\right\}$ has a maximal element. Let $z \in S_{2}$ be such that $R z$ is a maximal element of $T_{2}$. Thus, we have $w \in X_{1}, z \in X_{2}, x R \subseteq w R, R y \subseteq R z$ and $\neg(w R z)$.

We claim that $w$ and $z$ satisfy (Ti)(iii) and (Ti)(iv).

(Ti)(iii): Consider $u^{\prime} \in X_{1}$ such that $u^{\prime} \neq w$ and $w R \subseteq u^{\prime} R$. By (S), we have $u^{\prime} R \neq w R$ and so $w R \subsetneq u^{\prime} R$. Hence, $u^{\prime} R \notin T_{1}$ as $w R$ is a maximal element of $T_{1}$. Thus, $u^{\prime} \notin S_{1}$, but since $x R \subseteq w R \subseteq u^{\prime} R$, we must have $u^{\prime} R y$. Now $u^{\prime} R y$ implies $u^{\prime} \in R y \subseteq R z$ and so $u^{\prime} R z$.

(Ti)(iv): Consider $v^{\prime} \in X_{2}$ such that $v^{\prime} \neq z$ and $R z \subseteq R v^{\prime}$. By (S), we have $R z \neq R v^{\prime}$, and so $R v^{\prime} \notin T_{2}$ as $R z$ is a maximal element of $T_{2}$. Thus, $v^{\prime} \notin S_{2}$, but since $R y \subseteq R z \subseteq R v^{\prime}$, we must have $w R v^{\prime}$.

Gehrke's dual representation of perfect lattices via RS frames [11] gives, in the finite case, a dual representation of arbitrary finite lattices via finite RS 
frames. Our result above shows that the classes of finite RS frames and finite TiRS frames are the same, and so there is a dual representation of arbitrary finite lattices via finite TiRS frames. From our one-to-one correspondence between TiRS frames and TiRS graphs in Section 2, it follows now that there is a dual representation of arbitrary finite lattices via finite TiRS graphs. We know that when the considered finite lattices are distributive, the relation $E$ on the corresponding TiRS graphs becomes a partial order, and so in the distributive case, we obtain as a special case the well-known Birkhoff dual representation of finite distributive lattices via finite posets (see [7, Chapter 5]). Although Ploščica in [14] essentially obtained this dual representation of arbitrary finite lattices via finite graphs, our results (Section 2 and Proposition 3.1) enable us to characterise in an abstract way the finite graphs dually representing arbitrary finite lattices as exactly the finite TiRS graphs. We formulate this result explicitly as a corollary:

Corollary 3.2. There exists a dual representation of arbitrary finite lattices via finite TiRS graphs generalising the Birkhoff dual representation of finite distributive lattices via finite posets.

In this section we continue to generalise the descriptions of the completely join-irreducible and the completely meet-irreducible elements in the complete lattices $\mathrm{C}(\mathbf{X})$, which were presented for the graphs $\mathbf{X}=\left(\mathcal{L}^{\mathrm{mp}}(\mathbf{L}, \underline{\mathbf{2}}), E\right)$ in $[2]$ (cf. Proposition 5.5 and Corollary 5.6 there), to arbitrary reflexive reduced graphs $\mathbf{X}=(X, E)$.

Definition 3.3. Let $\mathbf{X}=(X, E)$ be a reflexive graph. For every element $x \in X$, the maps $J_{x}$ and $M_{x}$ are the partial maps from $X$ to 2 that, for $z \in X$, are given by:

$$
J_{x}(z)=\left\{\begin{array}{ll}
1 & \text { if } z E \subseteq x E, \\
0 & \text { if }(x, z) \notin E, \\
- & \text { otherwise }
\end{array} \quad \text { and } \quad M_{x}(z)= \begin{cases}1 \quad \text { if }(z, x) \notin E \\
0 \quad \text { if } E z \subseteq E x \\
-\quad \text { otherwise }\end{cases}\right.
$$

Proposition 3.4 ([2, Proposition 5.1]). Let $\mathbf{X}$ be a reflexive graph. Then the sets $\left\{J_{x} \mid x \in X\right\}$ and $\left\{M_{x} \mid x \in X\right\}$ are respectively join-dense and meet-dense in the lattice $\mathrm{C}(\mathbf{X})$.

When the graph is $\mathbf{X}=\mathrm{D}^{b}(\mathbf{L})$ for some bounded lattice $\mathbf{L}$, we also have

$$
J^{\infty}(\mathrm{C}(\mathbf{X}))=\left\{J_{x} \mid x \in X\right\} \quad \text { and } \quad M^{\infty}(\mathrm{C}(\mathbf{X}))=\left\{M_{y} \mid y \in X\right\},
$$

as was proven in [2] (cf. Proposition 5.5 and Corollary 5.6 there).

It turns out that these descriptions of completely join-irreducible and completely meet-irreducible elements of the lattice $\mathrm{C}\left(\mathrm{D}^{b}(\mathbf{L})\right)$ are not specific to the case $\mathbf{X}=\mathrm{D}^{b}(\mathbf{L})$, where $\mathbf{L} \in \mathcal{L}$. In fact, they apply to an arbitrary reflexive reduced graph $\mathbf{X}=(X, E)$. 
Proposition 3.5. If $\mathbf{X}$ is a reflexive reduced graph, then

$$
J^{\infty}(\mathrm{C}(\mathbf{X}))=\left\{J_{x} \mid x \in X\right\} \quad \text { and } \quad M^{\infty}(\mathrm{C}(\mathbf{X}))=\left\{M_{y} \mid y \in X\right\} .
$$

Proof. We prove that $J_{x}$ is completely join-irreducible. Assume that $J_{x}=$ $\bigvee\left\{\varphi_{i} \mid i \in I\right\}$, for some elements $\varphi_{i} \in \mathrm{C}(\mathbf{X})$ with $i \in I$. Since $\left\{J_{y} \mid y \in X\right\}$ is join-dense in the lattice $\mathrm{C}(\mathbf{X})$, we may assume that each $\varphi_{i}$ is $J_{x_{i}}$ for some $x_{i} \in X$. Suppose for a contradiction that $J_{x} \neq J_{x_{i}}$ for all $i \in I$. Then for every $i \in I, X \backslash x E=J_{x}^{-1}(0) \neq J_{x_{i}}^{-1}(0)=X \backslash x_{i} E$, and since $J_{x_{i}} \leqslant J_{x}$ and

$$
J_{x_{i}} \leqslant J_{x} \Rightarrow J_{x}^{-1}(0) \subseteq J_{x_{i}}^{-1}(0) \Rightarrow x_{i} E \subseteq x E,
$$

we must have $x_{i} E \subsetneq x E$. Therefore, by $(\mathrm{R})$, we have $\left(x_{i}, x\right) \notin E$ and so $J_{x_{i}}(x)=0$. Since $J_{x}=\bigvee\left\{J_{x_{i}} \mid i \in I\right\}$, we obtain $x \in \bigcap\left\{J_{x_{i}}^{-1}(0) \mid i \in I\right\}$ $=J_{x}^{-1}(0)$ (for the last equality, we use [2, Theorem 2.2]), which contradicts $J_{x}(x)=1$. Hence, $J_{x}$ must be one of the morphisms $J_{x_{i}}$, with $i \in I$.

Dually, one can argue that the element $M_{y}$ is completely meet-irreducible.

The fact that $J^{\infty}(\mathrm{C}(\mathbf{X})) \subseteq\left\{J_{x} \mid x \in X\right\}$ follows from Proposition 3.4 as every join-dense subset of a complete lattice contains the completely join-irreducible elements. The dual argument shows that $M^{\infty}(\mathrm{C}(\mathbf{X})) \subseteq$ $\left\{M_{y} \mid y \in X\right\}$.

The following corollary tells us that the lattice $\mathrm{C}(\mathbf{X})$ built from any reflexive reduced graph (in particular, from any TiRS graph) is a perfect lattice. It is an immediate consequence of Propositions 3.4 and 3.5.

Corollary 3.6. Let $\mathbf{X}=(X, E)$ be a reflexive reduced graph. The lattice $\mathrm{C}(\mathbf{X})$ is a perfect lattice. In fact, for every $\varphi \in \mathrm{C}(\mathbf{X})$, we have

$$
\varphi=\bigvee\left\{J_{x} \mid J_{x} \leqslant \varphi\right\} \quad \text { and } \varphi=\bigwedge\left\{M_{y} \mid \varphi \leqslant M_{y}\right\}
$$

and

$$
J^{\infty}(\mathrm{C}(\mathbf{X}))=\left\{J_{x} \mid x \in X\right\} \quad \text { and } \quad M^{\infty}(\mathrm{C}(\mathbf{X}))=\left\{M_{y} \mid y \in X\right\} .
$$

Let $\mathbf{X}$ be a reflexive reduced graph and consider the perfect lattice $\mathrm{C}(\mathbf{X})$ defined from $\mathbf{X}$. The RS frame dual to $\mathrm{C}(\mathbf{X})$ is the frame

$$
\mathbf{F}(\mathrm{C}(\mathbf{X}))=\left(J^{\infty}(\mathrm{C}(\mathbf{X})), M^{\infty}\left(\mathrm{C}(\mathbf{X}), R_{\mathrm{C}(\mathbf{X})}\right)\right.
$$

where $R_{\mathrm{C}(\mathbf{X})}$ is the restriction of the partial order $\leqslant$ of $\mathrm{C}(\mathbf{X})$ (see $[11$, Remark 2.13]), so $J_{x} R_{\mathrm{C}(\mathbf{X})} M_{y}$ iff $J_{x}^{-1}(1) \subseteq M_{y}^{-1}(1)$ iff $M_{y}^{-1}(0) \subseteq J_{x}^{-1}(0)$.

Proposition 3.7. Let $\mathbf{X}$ be a TiRS graph. The frame $\mathbf{F}(\mathrm{C}(\mathbf{X}))$ is a TiRS frame.

Proof. First observe that the following hold:

(i) $J_{x} R_{\mathrm{C}(\mathbf{X})} \subseteq J_{y} R_{\mathrm{C}(\mathbf{X})}$ iff $J_{y} \leqslant J_{x}$ iff $y E \subseteq x E$;

(ii) $R_{\mathrm{C}(\mathbf{X})} M_{x} \subseteq R_{\mathrm{C}(\mathbf{X})} M_{y}$ iff $M_{x} \leqslant M_{y}$ iff $E y \subseteq E x$;

(iii) $J_{x} R_{\mathrm{C}(\mathbf{X})} M_{y}$ iff $J_{x} \leqslant M_{y}$ iff $(x, y) \notin E$. 
Let $x, y \in X$ and assume that $\neg\left(J_{x} R_{\mathrm{C}(\mathbf{X})} M_{y}\right)$, that is, $(x, y) \in E$. Since $\mathbf{X}$ satisfies $(\mathrm{Ti})$, there exists $z \in X$ such that $z E \subseteq x E$ and $E z \subseteq E y$, so $J_{x} R_{\mathrm{C}(\mathbf{X})} \subseteq J_{z} R_{\mathrm{C}(\mathbf{X})}$ and $R_{\mathrm{C}(\mathbf{X})} M_{y} \subseteq R_{\mathrm{C}(\mathbf{X})} M_{z}$. If $u \in X$ is such that $J_{u} \neq J_{z}$ and $J_{z} R_{\mathrm{C}(\mathbf{X})} \subseteq J_{u} R_{\mathrm{C}(\mathbf{X})}$, then $u E \subsetneq z E$. But $\mathbf{X}$ satisfies $(\mathrm{S})$, and so we have $(u, z) \notin E$, that is, $J_{u} R_{\mathrm{C}(\mathbf{X})} M_{z}$. Similarly, we prove that $J_{z} R_{\mathrm{C}(\mathbf{X})} M_{v}$ whenever $v \in X$ satisfies $M_{v} \neq M_{z}$ and $R_{\mathrm{C}(\mathbf{X})} M_{z} \subseteq R_{\mathrm{C}(\mathbf{X})} M_{v}$. Thus, $\mathbf{F}(\mathrm{C}(\mathbf{X}))$ satisfies (Ti).

Lemma 3.8. Let $\mathbf{X}$ be a TiRS graph. The elements of the set $H_{\mathbf{F}(\mathrm{C}(\mathbf{X}))}$ are exactly the pairs $\left(J_{x}, M_{x}\right)$, with $x \in X$.

Proof. Observe that $\left(J_{x}, M_{y}\right) \in H_{\mathbf{F}(\mathrm{C}(\mathbf{X}))}$ iff

(i) $(x, y) \in E$;

(ii) for every $u \in X$, if $x E \neq u E$ and $u E \subseteq x E$ then $(u, y) \notin E$;

(iii) for every $v \in X$, if $E v \neq E y$ and $E v \subseteq E y$ then $(x, v) \notin E$.

Clearly, $\left(J_{x}, M_{x}\right) \in H_{\mathbf{F}(\mathrm{C}(\mathbf{X}))}$ for every $x \in X$ because $\mathbf{X}$ satisfies $(\mathrm{R})$. Suppose that $\left(J_{x}, M_{y}\right) \in H_{\mathbf{F}(\mathrm{C}(\mathbf{X}))}$. Since $(x, y) \in E$ and $\mathbf{X}$ satisfies (Ti), there exists $z \in X$ such that $z E \subseteq x E$ and $E z \subseteq E y$. Since $(x, z),(z, y) \in E$, we must have $x E=z E$ and $E y=E z$. Hence, $J_{x}=J_{z}$ and $M_{y}=M_{z}$, so $\left(J_{x}, M_{y}\right)=\left(J_{z}, M_{z}\right)$.

Theorem 3.9. Let $\mathbf{X}$ be a TiRS graph. We have that

$$
\operatorname{gr}(\mathbf{F}(\mathrm{C}(\mathbf{X}))) \simeq \mathbf{X} \quad \text { and } \quad \rho(\mathbf{X}) \simeq \mathbf{F}(\mathrm{C}(\mathbf{X}))
$$

Proof. By Lemma 3.8, the vertex set $H$ of $\operatorname{gr}(\mathbf{F}(\mathrm{C}(\mathbf{X})))$ is $\left\{\left(J_{x}, M_{x}\right) \mid x \in X\right\}$. Consider the onto map $\phi: \mathbf{X} \rightarrow \operatorname{gr}(\mathbf{F}(\mathrm{C}(\mathbf{X})))$ given by $\phi(x)=\left(J_{x}, M_{x}\right)$. Since $\mathbf{X}$ satisfies $(\mathrm{S})$, if $x \neq y$, then either $x E \neq y E$ or $E x \neq E y$. Without loss of generality, suppose that $x E \neq y E$. Then there exists $z \in X$ such that $(x, z) \in E$ but $(y, z) \notin E$, which implies that either $z \notin \operatorname{dom} J_{x}$ or $J_{x}(z)=1$ but $z \in \operatorname{dom} J_{y}$ and $J_{y}(z)=0$. Hence, $J_{x} \neq J_{y}$, and so $\phi(x) \neq \phi(y)$. Thus, $\phi$ is one-to-one.

Finally, we show that $(x, y) \in E$ iff $(\phi(x), \phi(y)) \in K$. Recall that

$$
(\phi(x), \phi(y)) \in K \Longleftrightarrow\left(J_{x}, M_{y}\right) \notin R \Longleftrightarrow J_{x} \nless M_{y} \Longleftrightarrow J_{x}^{-1}(1) \nsubseteq M_{y}^{-1}(1) .
$$

Lastly, observe that $J_{x} \nless M_{y}$ iff $(x, y) \in E$.

Thus, we have that $\mathbf{X} \simeq \operatorname{gr}(\mathbf{F}(\mathrm{C}(\mathbf{X})))$. Theorem 2.13 and Proposition 3.7 yield $\rho(\mathbf{X}) \simeq \mathbf{F}(\mathrm{C}(\mathbf{X}))$.

For a bounded lattice $\mathbf{L}$, recall that the canonical extension $\mathbf{L}^{\delta}$ of $\mathbf{L}$ can be taken as $\mathrm{C}(\mathbf{X})$, where $\mathbf{X}$ is the TiRS graph $\mathrm{D}^{\mathrm{b}}(\mathbf{L})$. The following result is an immediate consequence of the previous theorem.

Corollary 3.10. Let $\mathbf{L}$ be a bounded lattice and let $\mathbf{L}^{\delta}$ be its canonical extension. The dual RS frame $\mathbf{F}\left(\mathbf{L}^{\delta}\right)$ of $\mathbf{L}^{\delta}$ is TiRS and

$$
\operatorname{gr}\left(\mathbf{F}\left(\mathbf{L}^{\delta}\right)\right) \simeq \mathrm{D}^{b}(\mathbf{L}) \text { and } \quad \rho\left(\mathrm{D}^{b}(\mathbf{L})\right) \simeq \mathbf{F}\left(\mathbf{L}^{\delta}\right) .
$$


We have shown that the RS frame associated to the canonical extension is always TiRS. Due to this and the correspondence between RS frames and perfect lattices, one could ask whether also conversely, every TiRS frame corresponds to a perfect lattice that is some canonical extension. So, could it be that the perfect lattices corresponding to TiRS frames are exactly those that are the canonical extensions? We know again from the distributive case that this question can be answered negatively. A non-representable poset is a TiRS graph, and hence its corresponding frame is also TiRS. However, it does not correspond to the canonical extension of any bounded (distributive) lattice. Therefore, our second open problem is posed as a slightly different question:

Problem 2. Consider the perfect lattice that corresponds to a TiRS frame. In addition to being perfect, what additional properties of the complete lattice arise as a result of it coming from an $R S$ frame that also satisfies (Ti)?

Finally, we are going to show that Ploščica's dual representation of bounded lattices and the dual representation between RS frames and perfect lattices can be used in tandem to provide a new construction of the canonical extension for an arbitrary bounded lattice.

Following Gehrke [11], for an $\mathrm{RS}$ frame $\mathbf{F}=(X, Y, R)$, a Galois connection between $\wp(X)$ and $\wp(Y)$ is considered, where the maps $(\cdot)^{R}$ and ${ }^{R}(\cdot)$ are defined as follows: for $A \subseteq X$ and $B \subseteq Y$,

$$
(A)^{R}=\{y \in Y \mid \forall a \in A, a R y\} \quad \text { and } \quad R(B)=\{x \in X \mid \forall b \in B, x R b\} .
$$

The perfect lattice of Galois-closed sets associated to $\mathbf{F}$ is denoted by $\mathrm{G}(\mathbf{F})$. For the dual RS frame $\mathbf{F}(\mathbf{C})=\left(J^{\infty}(\mathbf{C}), M^{\infty}(\mathbf{C}), \leqslant\right)$ of a perfect lattice $\mathbf{C}$, the results of [11] show that $\mathbf{C}$ is isomorphic to $\mathrm{G}(\mathbf{F}(\mathbf{C}))$. Hence, considering $\mathbf{C}=\mathbf{L}^{\delta}$ and using Corollary 3.10, we immediately obtain our final result.

Corollary 3.11. Let $\mathbf{L}$ be a bounded lattice and $\mathbf{X}=\mathrm{D}^{b}(\mathbf{L})$ be its dual TiRS graph. Let $\rho(\mathbf{X})$ be the frame associated to $\mathbf{X}$ and $\mathrm{G}(\rho(\mathbf{X}))$ its corresponding perfect lattice of Galois-closed sets. Then the lattice $\mathrm{G}(\rho(\mathbf{X}))$ is the canonical extension of $\mathbf{L}$.

We remark that an alternative (and in fact more straightforward) way of proving the previous result is as follows. We only have to show that the frames $\rho\left(\mathrm{D}^{b}(\mathbf{L})\right)$ and $\mathbf{F}\left(\mathbf{C}\left(\mathrm{D}^{b}(\mathbf{L})\right)\right)$ are isomorphic. However, one can easily see that $\rho\left(\mathrm{D}^{\mathrm{b}}(\mathbf{L})\right)=\left(X_{1}, X_{2}, R\right)$, where $X_{1}$ is the set of all filters $x^{-1}(1)$ and $X_{2}$ is the set of all ideals $x^{-1}(0)$ for $x \in \mathrm{D}^{b}(\mathbf{L})$. Further, one can check that we have $\mathbf{F}\left(\mathbf{C}\left(\mathrm{D}^{b}(\mathbf{L})\right)\right)=\left(Y_{1}, Y_{2}, \leqslant\right)$ with $Y_{1}=\left\{J_{x} \mid x \in \mathrm{D}^{b}(\mathbf{L})\right\}$ and $Y_{2}=\left\{M_{y} \mid y \in \mathrm{D}^{b}(\mathbf{L})\right\}$. So, we have obvious assignments $J_{x} \mapsto x^{-1}(1)$ and $M_{y} \mapsto y^{-1}(0)$, and it is almost straightforward to verify that these assignments define a frame isomorphism. (We are thankful to the referee for suggesting this remark.) 


\section{REFERENCES}

[1] Clark D.M., Davey, B.A.: Natural Dualities for the Working Algebraist. Cambridge University Press (1998)

[2] Craig, A.P.K., Haviar, M.: Reconciliation of approaches to the construction of canonical extensions of bounded lattices. Math. Slovaca 64, 1335-1356 (2014)

[3] Craig, A.P.K., Haviar, M., Priestley, H.A.: A fresh perspective on canonical extensions for bounded lattices. Appl. Categ. Structures 20, 725-749 (2013)

[4] Davey, B.A.: A note on representable posets. Algebra Universalis 3, 345-347 (1973)

[5] Davey, B.A., Gouveia, M.J., Haviar, M., Priestley, H.A.: Natural extensions and profinite completions of algebras. Algebra Universalis 66, 331-336 (2011)

[6] Davey, B.A., Haviar, M., Priestley, H.A.: Boolean topological distributive lattices and canonical extensions. Appl. Categ. Structures 15, 225-241 (2007)

[7] Davey, B.A., Priestley, H.A.: Introduction to Lattices and Order, 2nd edn. Cambridge University Press (2002)

[8] Davey, B.A., Priestley, H.A.: A topological approach to canonical extensions in finitely generated varieties of lattice-based algebras. Topology Appl. 158, 1724-1731 (2011)

[9] Davey, B.A., Priestley, H.A.: Canonical extensions and discrete dualities for finitely generated varieties of lattice-based algebras. Studia Logica 100, 137-161 (2012)

[10] Dunne, J.M., Gehrke, M., Palmigiano, A.: Canonical extensions and relational completeness of some substructural logics. J. Symbolic Logic 70, 713-740 (2005)

[11] Gehrke, M.: Generalized Kripke frames. Studia Logica 84, 241-275 (2006)

[12] Gehrke, M., Harding, J.: Bounded lattice expansions. J. Algebra 238, 345-371 (2001)

[13] Gehrke, M., Vosmaer, J.: A view of canonical extension. In: Proceedings of the Eighth International Tbilisi Symposium, TbiLLC, 2009. Lecture Notes in Computer Science vol. 6618, pp. 77-100. Logic, Language and Computation (Tbilisi, 2011)

[14] Ploščica, M.: A natural representation of bounded lattices. Tatra Mountains Math. Publ. 5, 75-88 (1995)

[15] Urquhart, A.: A topological representation theory for lattices. Algebra Universalis 8, $45-58(1978)$

\section{AndRew P.K. Craig}

Department of Pure and Applied Mathematics, University of Johannesburg, PO Box 524, Auckland Park, 2006, South Africa

e-mail: acraig@uj.ac.za

\section{Maria J. Gouveia}

Faculdade de Ciências da Universidade de Lisboa \& CAUL, P-1749-016 Lisboa, Portugal

e-mail: mjgouveia@fc.ul.pt

\section{Miroslav Haviar}

Faculty of Natural Sciences, M. Bel University, Tajovského 40, 97401 Banská Bystrica, Slovakia

e-mail: miroslav.haviar@umb.sk 